\title{
Der Antiferromagnetismus des Ferritins bei Messungen der magnetischen Suszeptibilität im Temperaturbereich von 4,2 bis $300^{\circ} \mathrm{K}$
}

\author{
Georg Schoffa*, Karlsruhe \\ Institut für Strahlenforschung der Universität Kiel in Lübeck (Direktor Prof. Dr. H. Schreiber)
}

(Z. Naturforschg. 20 b, 167-172 [1965] ; eingegangen am 7. November 1964)

\begin{abstract}
Measurements of magnetic susceptibility in the temperature range $4.2-300{ }^{\circ} \mathrm{K}$ show that ferritin is antiferromagnetic with a Néel temperature of $20^{\circ} \pm 3{ }^{\circ} \mathrm{K}$. The theory of J. S. Smart for antiferromagnetic exchange between iron atoms clustered in groups of two ("isolated clusters") gives the best agreement between theoretical and experimental values. The antiferromagnetic exchange constant is $J / k=-4.8\left({ }^{\circ} \mathrm{K}\right)$. Reduced magnetic moment for $\mu_{\mathrm{eff}}=3.85 \mu_{\mathrm{B}}$ is due to the transfer of two electrons from oxygen atoms to ferric atoms caused on the cation-anion-cation superexchange. Some models of superexchange are discussed. Antiferromagnetism and superexchange are possibly caused on the cubic magnetic structure of iron-oxygen micelles in ferritin.
\end{abstract}

Ferritin ist ein besonders in Milz, Leber und Knochenmark angereichert vorkommendes Eisenprotein. Eine seiner wichtigsten Aufgaben ist es, Eisen zu speichern und der Hämoglobinsynthese laufend zur Verfügung zu stellen. Das Mol.-Gew. des Ferritins beträgt etwa 460000 . Der Eisengehalt liegt zwischen 19 und 23 Gew.-Prozent. Ferritin verfügt also über eine überraschend gute Speicherungskapazität für Eisen. Die Struktur seines Eiweißkörpers, des sogenannten Apoferritins, konnte trotz mehrfacher elektronenmikroskopischer ${ }^{1-3}$ und röntgenstrukturanalytischer ${ }^{4,5}$ Untersuchungen nur teilweise geklärt werden. Das Eisen im Ferritin bildet ca. $50 \AA$ große, vom Eiweißmolekül umhüllte Mizellen, die wieder in kleinere, ca. $8 \AA$ große Einheiten unterteilt sind ${ }^{6}$. Die chemische Struktur des Eisenkomplexes des Ferritins ist nicht genau bekannt. Michaelis, Coryell und Granick ${ }^{7,8}$ schlugen die Strukturen $\mathrm{Fe}-\mathrm{O}-\mathrm{Fe}$ oder $\mathrm{Fe}\left\langle\mathrm{O}{ }_{\mathrm{O}}^{\mathrm{O}}\right\rangle \mathrm{Fe}$ vor; als Summenformel wird

$$
(\mathrm{FeO} \cdot \mathrm{OH})_{8}\left(\mathrm{FeO} \cdot \mathrm{OPO}_{3} \mathrm{H}_{2}\right)
$$

angenommen ${ }^{9}$.

* Zur Zeit Physikalisches Institut der T.H. Karlsruhe (Direktor Prof. Dr. W. BuckeL).

1 J. L. Farrant, Biochim. biophysica Acta [Amsterdam] 13, 569 [1954].

2 R. W. G. Wускоғғ, Biochim. biophysica Acta [Amsterdam] 25, 263 [1957].

3 A. R. Muir, Quart. J. exp. Physiol.. 45, 192 [1960].

4 P. A. Harrison, J. molecular Biol. 1, 69 [1959].

5 I. Fankuchen, J. biol. Chemistry 150, 57 [1943].
Bei dieser Sachlage sind Messungen der statischen magnetischen Suszeptibilität von besonderem Interesse, da aus ihnen Einzelheiten über die chemische Bindung des Eisens im Ferritin ermittelt werden können. Die ersten Messungen der magnetischen Suszeptibilität wurden bald nach der Entdeckung und Isolierung des Ferritins von Michaelis, Coryell und Granick ${ }^{7}$ im Temperaturbereich von $275^{\circ}$ bis $301{ }^{\circ} \mathrm{K}$ ausgeführt. Eine gefundene geringfügige Abweichung vom $\mathrm{Cu}$ ri e-Gesetz wurde als Streuung der Meßwerte gedeutet. Unter Annahme der Gültigkeit des Curie-Gesetzes wurde das magnetische Moment $\mu_{\text {eff }}=3,81 \mu_{\mathrm{B}}$ erhalten. Bayer und HausSER ${ }^{10}$ fanden später $\mu_{\text {eff }}=3,84 \mu_{\mathrm{B}}$ und bei $293{ }^{\circ} \mathrm{K}$, $200{ }^{\circ} \mathrm{K}$ und $90^{\circ} \mathrm{K}$ stellten sie eine deutliche Abweichung der Temperaturabhängigkeit der magnetischen Suszeptibilität vom C u r i e - Gesetz fest.

In der vorliegenden Arbeit wird über Messungen der magnetischen Suszeptibilität im Temperaturbereich von $4,2^{\circ}$ bis $300{ }^{\circ} \mathrm{K}$ berichtet. Die Erweiterung des Meßbereiches bis zur Temperatur des flüssigen Heliums ermöglichte eine Klärung des magnetischen Zustandes des Ferritins und führte zu einigen neuen Erkenntnissen über die chemische Bindung und Struktur des Eisens im Ferritin.

6 G. W. Richter, J. exp. Medicine 106, 203 [1957].

7 L. Michaelis, Ch. D. Coryell u. S. Granick, J. biol. Chemistry 148, 463 [1943].

8 S. Granick, Chem. Reviews 38, 379 [1946].

9 S. Granick u. P. F. Hahn, J. biol. Chemistry 155, 661 [1944].

10 E. Bayer u. K. H. Hausser, Experientia [Basel] 11/7, 254 [1955]. 


\section{Methodik}

Die verwendete Apparatur zur Messung der magnetischen Suszeptibilität im Temperaturbereich von $4,2^{\circ}$ bis $300{ }^{\circ} \mathrm{K}$ ist in der Abb. 1 zu sehen. Sie bestand aus einem niederohmigen Elektromagneten der Fa. Newport, einem Netzgerät, das aus $50 \mathrm{~Hz}$-Netzspannung Gleichspannung erzeugte, diese stabilisierte und die

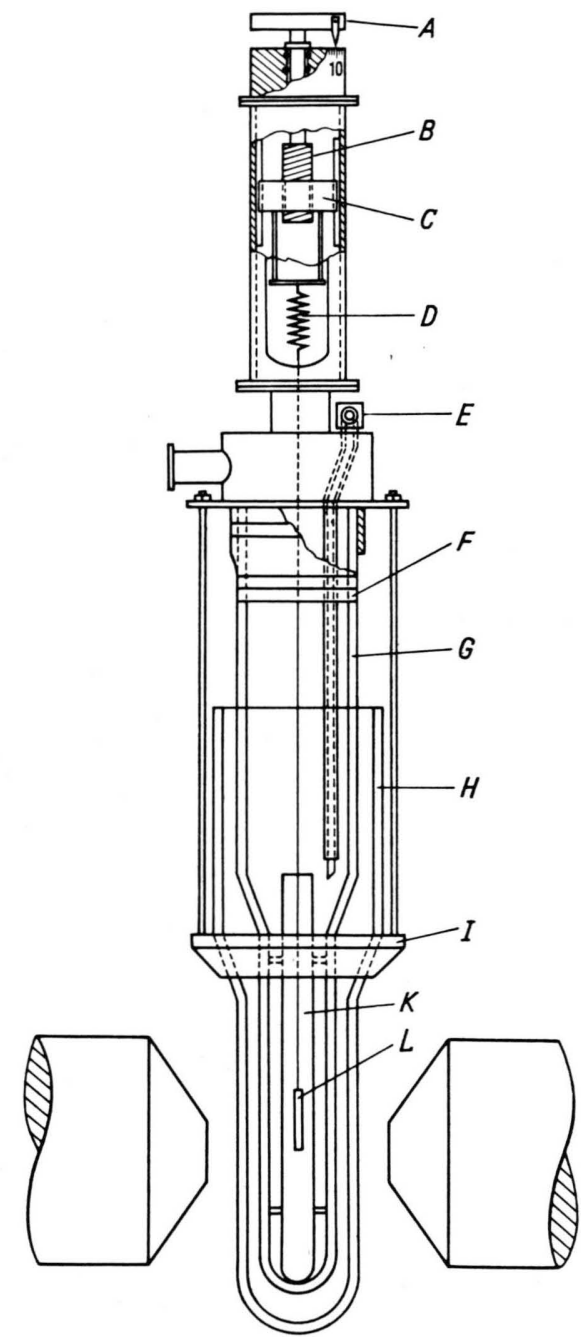

Abb. 1. Apparatur zur Messung der magnetischen Suszeptibilität im Temperaturbereich von $4,2^{\circ}$ bis $300^{\circ} \mathrm{K}$. A - Drehknopf, B - Schraubspindel, C - Kolben, D - Phosphorbronzefeder, E - Heliumheber, F - Gummimanschetten mit Schlauchbinden, G - inneres D e w a r - Gefäß, H - äußeres D e w a r-Gefäß, I - Halterung für das äußere D e w a r Gefäß, K - Reagenzröhrchen, L - Probe.

stufenlose Einstellung des durch die Spulen des Elektromagneten fließenden Stromes im Bereich von 0,1 bis 10 A erlaubte, und schließlich aus der Meßeinrichtung zur Messung der magnetichen Suszeptibilität. Die durch Gleichstrom im Magneten erzeugte Magnetfeldstärke wurde am Ort der Probe mit Hilfe der Protonenresonanz gemessen und betrug wegen des durch zwei D e w a r-Gefäße bedingten großen Polschuhabstandes maximal ca. $5000 \mathrm{Gauß}$. Die Messungen wurden bei 2600,3600 und $4600 \mathrm{Gau}$ ausgeführt, um eventuelle Verunreinigungen der Meßsubstanz durch ferromagnetische Stoffe nicht zu übersehen.

Das Kernstück der Einrichtung zur Messung der magnetischen Suszeptibilität war eine Spiralfeder aus Phosphorbronzedraht, an welche die Meßprobe mit Hilfe eines ca. $100 \mathrm{~cm}$ langen und $10 \mu$ starken Wolframfadens angehängt wurde. Die Feder war an einem Metallkolben befestigt und konnte mittels einer Schraubspindel, die von außen betätigt wurde, hoch- oder heruntergeschraubt werden, ohne daß sie sich hierbei um ihre eigene Achse drehte. Die Feder mit der Spindel und Probe befanden sich in einem Zylinder, welcher vakuumdicht an das innere D e w a r - Gefäß angeschlossen wurde. Die Achse der Spindel wurde vakuumdicht nach außen geführt. Durch ein Fenster im Zylinder konnte das Ende des Wolframfadens bzw. der Feder mit einem Meßmikroskop beobachtet werden. Der Verbindungsteil zwischen innerem D e w a r-Gefäß und Zylinder enthielt Durchführungen für das Thermoelement und den Thermistor, den Abpumpstutzen und den Heber für das flüssige Helium. Um dessen schnelles Verdampfen zu verhindern, befand sich das innere D e w a r-Gefäß in einem äußeren, mit flüssiger Luft gefüllten zweiten Gefäß. Vor jeder Messung wurde das innere Gefäß bis auf einen Druck von $10^{-2}$ Torr evakuiert und dann das Heliumgas eingelassen. Nach Füllung des äußeren Gefäßes mit flüssiger Luft wurde ca. 30 Min. gewartet, während welcher Zeit sich die Probe auf $90{ }^{\circ} \mathrm{K}$ abkühlte. Dann wurde das Helium-Vorratsgefäß an den Heber angeschlossen und durch Ansaugen mit einer heliumdichten Pumpe flüssiges Helium in das innere D e w a r-Gefäß eingefüllt. Das Helium umspülte dabei ein Reagenzröhrchen, in dem die Meßprobe frei hing.

Die Temperaturmessung am Ort der Probe erfolgte im Temperaturbereich zwischen $4,2^{\circ}$ und $20^{\circ} \mathrm{K}$ durch Messen des Widerstandes eines Thermistors und im Bereich von $20^{\circ}$ bis $300{ }^{\circ} \mathrm{K}$ durch Messen des Widerstandes einer Spule aus Platin, in deren Innenraum sich die Probe befand. Die Temperatur von $4,2{ }^{\circ} \mathrm{K}$ konnte lange Zeit genau eingehalten werden. Höhere Temperaturen wurden durch Erwärmen der Probe nach dem Verdampfen des Heliums erhalten; es dauerte etwa eine Stde., bis die Probe so auf Zimmertemperatur gebracht werden konnte. Obwohl der Thermistor bzw. der Platindraht unmittelbar an der Meßprobe anlagen, entsprach ihre Temperatur nicht genau der Temperatur der Meßprobe. Die Eichung der Anordnung erfolgte daher so, daß zunächst Suszeptibilitäts-Bestimmungen an einer $\mathrm{CuSO}_{4} \cdot 5 \mathrm{H}_{2} \mathrm{O}$-Probe durchgeführt wurden, deren $\mathrm{Ab}$ messungen mit der später zur Messung kommenden Ferritin-Probe übereinstimmten. Die Kupfersulfat-Probe befand sich selbstverständlich auch an dem genau festgelegten Ort, an den später die Ferritin-Probe kam. Die Temperaturabhängigkeit der magnetischen Suszeptibilität des $\mathrm{CuSO}_{4} \cdot 5 \mathrm{H}_{2} \mathrm{O}$ ist von verschiedenen Autoren 
hinreichend genau bestimmt worden mit dem übereinstimmenden Resultat, daß bis $4,2{ }^{\circ} \mathrm{K}$ das $\mathrm{C} \mathrm{u} \mathrm{ri} \mathrm{e-Ge-}$ setz erfüllt ist. Über diese bekannten Suszeptibilitätswerte des $\mathrm{CuSO}_{4} \cdot 5 \mathrm{H}_{2} \mathrm{O}$ konnten die angezeigten Widerstände des Thermistors bzw. des Platindrahtes in Verbindung mit der Temperatur am Probenort gebracht werden. Die Genauigkeit der so ermöglichten Temperaturbestimmungen war bei tieferen Temperaturen mit $\pm 3^{\circ}$ geringer als bei höheren Temperaturen, da bei tieferen Temperaturen eine Konstanz bzw. Gleichmäßigkeit der Temperaturverteilung über die gesamte Probe, infolge des größeren Temperaturgradienten zur Umgebung hin, nicht in dem gleichen Maße gewährleistet war wie bei höheren Temperaturen, bei denen eine Genauigkeit der Temperaturbestimmung von $\pm 2^{\circ}$ erreicht wurde.

Die Messungen selbst wurden folgendermaßen ausgeführt: Zunächst wurde das obere Ende des Aufhängefadens mit einem Meßmikroskop beobachtet und auf die Nullmarke justiert. Beim Einschalten des Magnetes verlängerte sich die Feder, weil das paramagnetische Ferritin in das Magnetfeld hineingezogen wurde. Die Feder mit Faden und Probe wurde nun mit Hilfe der Spindel solange hochgedreht, bis im Meßmikroskop die Beobachtungsmarke wieder am gleichen Ort wie vor dem Einschalten des Magnetfeldes zu sehen war. Die Zahl der Umdrehungen der Schraubspindel ist proportional der Verlängerung der Feder und damit auch der vom Magnetfeld auf die Probe ausgeübten Auziehungskraft. Die auf Eisen bezogene molare magnetische Suszeptibilität wurde wie üblich berechnet (vgl. dazu Schoffa 1. c. ${ }^{11}$ ). Die Gerätekonstante wurde durch Messungen an Proben aus

$\mathrm{HgCo}(\mathrm{CNS})_{4}$ und $\mathrm{Fe}_{2} \mathrm{SO}_{4}\left(\mathrm{NH}_{4}\right)_{2} \mathrm{SO}_{4} \cdot 6 \mathrm{H}_{2} \mathrm{O}$, deren magnetische Suszeptibilitäten genau bekannt sind ${ }^{11}$, ermittelt.

Das von der Firma Nutritional Biochemical Corporation bezogene Ferritin war nach Angaben der Firma nach Granick ${ }^{12}$ aus der Pferdemilz isoliert und durch zweimalige Umkristallisation gereinigt. Nach der Bezeichnung von P. A. HARRISON ${ }^{4}$ handelt es sich hier um Ferritin A. Dies konnte auch durch den Nachweis der Anisotropie der Elektronenresonanz-Spektren bestätigt werden. Bei der Berechnung der magnetischen Suszeptibilität wurde der analytisch gewonnene Wert für den Eisengehalt ${ }^{13}$ benutzt. Das zur Stabilisierung des Ferritins zugefügte Cadmiumsulfat ist diamagnetisch und brauchte daher nur bei der Korrektur hinsichtlich des Diamagnetismus berücksichtigt zu werden. Wegen der hohen Konzentration des Eisens war diese Korrektur gering.

\section{Ergebnisse und Diskussion}

Die auf Eisen bezogene statische magnetische Suszeptibilität des Ferritins betrug bei Zimmertempera-

11 G. Schoffa, Elektronenspinresonanz in der Biologie, BraunVerlag, Karlsruhe 1964, Seite 284.

12 S. Granick, J. biol. Chemistry 147, 19 [1943]. tur $\chi_{\mathrm{Fe}, 20^{\circ}}=6050 \cdot 10^{-6}$. Unter der Annahme, daß das C u r i e - Gesetz gültig ist, erhält man daraus das magnetische Moment zu

$$
\mu_{\text {eff }}=2,829 \sqrt{\chi_{\mathrm{Fe}} \cdot 293^{\circ}} \cdot \mu_{\mathrm{B}}=3,85 \mu_{\mathrm{B}} .
$$

Dieser Wert steht in guter Übereinstimmung mit allen bisher erhaltenen Werten.

Ein ungewöhnliches Verhalten zeigte die Temperaturabhängigkeit der magnetischen Suszeptibilität des Ferritins. Während bei normalen paramagnetischen Stoffen die magnetische Suszeptibilität mit abnehmenden Temperaturen hyperbolisch zunimmt $(\chi=C / T)$, sieht man auf der Abb. 2 zunächst eine Zunahme und dann ab $20{ }^{\circ} \mathrm{K}$ eine starke $\mathrm{Ab}$ -

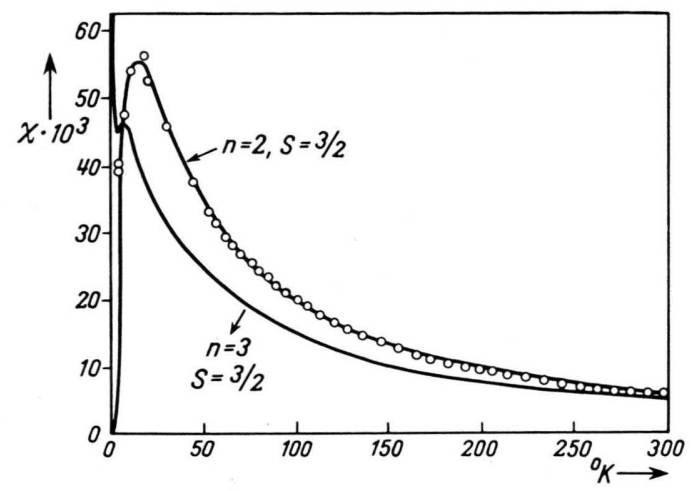

Abb. 2. Magnetische Suszeptibilität des Ferritins im Temperaturbereich von $4,2^{\circ}$ bis $300^{\circ} \mathrm{K}$. Die ausgezogenen Linien sind die theoretischen Kurven für die Temperaturabhängigkeit der magnetischen Suszeptibilität nach SMART ${ }^{14}$ für zwei $(n=2)$, bzw. drei $(n=3)$ miteinander antiferromagnetisch wechselwirkende Ionen mit $S=3 / 2$ und $J / k=-4,8{ }^{\circ} \mathrm{K}$.

nahme der magnetischen Suszeptibilität. Eine solche Temperaturabhängigkeit des Paramagnetismus ist typisch für Stoffe mit antiferromagnetischer Wechselwirkung zwischen den ungepaarten Elektronen. Die Temperatur, unterhalb welcher die magnetische Suszeptibilität abnimmt, heißt antiferromagnetische Curie-Temperatur in Analogie zur ferromagnetischen Curie-Temperatur. Sie liegt bei Ferritin bei $20^{\circ} \pm 3{ }^{\circ} \mathrm{K}$.

Die Abnahme des Paramagnetismus unterhalb der C u r i e - Temperatur beruht darauf, daß infolge der Austauschkräfte zwischen den Spins der benachbarten Atome die Antiparallelstellung der Spins stark begünstigt wird. Diese Erscheinung findet man mindestens so häufig wie die Parallelstellung der Spins

13 C. BÉnÉzech u. B. M. de Bornier, Bull. Soc. Chim. biol. 38, 661 [1956]. 
bei ferromagnetischen Stoffen. Ferritin ist jedoch die erste biologische Substanz, bei der Antiferromagnetismus bekannt geworden ist.

Die für Ferromagnetismus und Antiferromagnetismus verantwortlichen Austauschkräfte sind quantenmechanische Effekte, die der klassischen Physik unbekannt sind. Elektrische oder magnetische DipolDipol-Wechselwirkungen oder andere innermolekulare Kräfte können den Antiferromagnetismus nicht erklären. Bei Ferritin sind ähnlich wie bei den meisten anderen Antiferromagnetika die magnetischen Dipole durch diamagnetische Ionen voneinander räumlich getrennt. Die Wechselwirkung der magnetischen Dipole über diese nichtmagnetischen Koppelionen beruht auf dem Effekt der indirekten Wechselwirkung („superexchange“). Sie ist heute weitgehend erforscht und kann auch quantenmechanisch beschrieben werden.

Im folgenden soll auf die Zusammenhänge zwischen der magnetischen Suszeptibilität und den Austauschkräften eingegangen werden. Die Austauschwechselwirkung zwischen $\operatorname{dem} i$-ten und $\operatorname{dem} j$-ten Atom ist nach $\mathrm{He}$ is e $\mathrm{n}$ be $\mathrm{r}$ g durch die Beziehung

$$
W_{\mathrm{ij}}=-2 J_{\mathrm{ij}} S_{\mathrm{i}} S_{\mathrm{j}}
$$

gegeben, wobei $J_{\mathrm{ij}}$ das Austauschintegral und $S_{\mathrm{i}}$ und $S_{\mathrm{j}}$ die Spinoperatoren der Atome $i$ und $j$ sind. Im Falle der ferromagnetischen Wechselwirkung ist $J$ positiv, bei antiferromagnetischer Wechselwirkung dagegen negativ. Die Anwendung der Gleichung auf konkrete Fälle stößt auf große Schwierigkeiten, sogar wenn man nur Wechselwirkungen zwischen unmittelbaren Nachbarn berücksichtigt. Die Behandlung des Problems vereinfacht sich aber, wenn man annimmt, daß statt der Wechselwirkungen zwischen mehreren Eisenatomen lediglich eine Wechselwirkung zwischen zwei Eisenatomen zu berücksichtigen ist. Die Eisenatom-Paare sind wie in eine diamagnetische Matrix eingelagert anzusehen (,isolated clusters"). Die theoretischen Kurven für die magnetische Suszeptibilität in Abhängigkeit von der Spinquantenzahl, von der Zahl der in Wechselwirkung stehenden Atome $(n=2,3,4)$ und von der Größe der Austauschwechselwirkung $J$ in solchen „isolated cluster" gab neulich Smart an. Seine Berechnung gilt nur für Atome mit gleichen Spinquantenzahlen $S$ und einem Austauschparameter $J$. Der $\mathrm{Hamilt}$ on-Operator für dieses vereinfachte System ist

$$
\mathcal{H}=-2 \underset{i<j}{J \sum_{i<j}} S_{\mathrm{i}} \cdot S_{\mathrm{j}}-g \mu_{\mathrm{B}} S_{\mathrm{z}}{ }^{\prime} H
$$

wobei $S_{\mathrm{z}}{ }^{\prime}$ den Operator der $z$-Komponente des Gesamtspins, $g$ den Landé-Faktor, $\mu_{\mathrm{B}}$ das Boh r sche Magneton und $H$ das äußere Magnetfeld bedeuten. Die angenäherte Lösung dieser Funktion ist bei $\mathrm{SMART}^{14}$ angegeben. Im wesentlichen ist das Ergebnis dieser Theorie, daß z. B. für $S=3 / 2$ und $n=2$ bei $T=0$ die Atome sich im Zustand $S^{\prime}=0$ befinden und damit kein magnetisches Moment besitzen. Mit zunehmender Temperatur gelangen immer mehr Atome auf höher liegende magnetische $\mathrm{Zu}$ stände und der Paramagnetismus nimmt zu. Dies geschieht solange, bis die Temperatur der Probe so hohe Werte erreicht, daß die Spins im statistischen Mittel eine so starke Desorientierung erfahren, daß die magnetische Suszeptibilität wieder, aber nun nach dem C u r i e - Gesetz abnimmt. Die beste Übereinstimmung zwischen den theoretischen und den von uns gefundenen Werten für die magnetische Suszeptibilität erhält man, wenn man für $n=2$ und $S=3 / 2$ die antiferromagnetische Wechselwirkung $J / k=-4,8\left({ }^{\circ} \mathrm{K}\right)$ einsetzt. Die theoretischen Kurven für $S=5 / 2$ und $S=1 / 2$ könnte man nur unter Annahme recht unwahrscheinlicher Werte für $J$ mit experimentellen Werten in Übereinstimmung bringen. Wegen $\mu_{\mathrm{eff}}=3,85 \mu_{\mathrm{B}}$ sind beide Spinquantenzahlen unwahrscheinlich. Eine Wechselwirkung von drei Eisenionen untereinander würde zu einer völlig anderen Temperaturabhängigkeit der magnetischen Suszeptibilität führen. Die Bestimmung der Austauschkonstanten $J$ ist mit einigen Fehlern behaftet, da bei einer exakten Behandlung des Problems noch Wechselwirkungen mit weiteren Nachbarn berücksichtigt werden müßten.

Der Grundzustand des freien $\mathrm{Fe}^{3 \oplus}$.Ions ist ${ }^{6} S$ mit $S=5 / 2$. Das zu erwartende magnetische Moment wäre $\mu=5,92 \mu_{\mathrm{B}}$. Das experimentell von uns und anderen Autoren gefundene magnetische Moment von $\mu_{\text {eff }}=3,85 \mu_{\mathrm{B}}$ entspricht aber fast genau der Spinquantenzahl $S=3 / 2$. Wenn es sich bei Ferritin um einen normalen $\mathrm{Fe}^{3 \oplus}$-Komplex handeln würde, wäre aber $S=3 / 2$ theoretisch kaum zu verstehen. Bei antiferromagnetischen Stoffen sind jedoch im Rahmen der indirekten Wechselwirkung („superexchange“) Verschiebungen der Elektronen über ein unmagnetisches Kopplungsion möglich. Die quantentheoretische Begründung für diesen Effekt gab zuerst Kramers ${ }^{15}$, Anderson ${ }^{16}$ erweiterte dann die Theorie der indirekten Wechselwirkung und gab für

14 J. S. Smart, Magnetism, Vol. III, Ed. G. T. Rado u. H. Suhl, Academic Press, New York 1963, Seite 69. 
den Fall $\mathrm{Me}^{2 \oplus} \mathrm{O}^{2 \ominus} \mathrm{Me}^{2 \oplus}$ die anschauliche Deutung, daß durch eine indirekte Wechselwirkung dieser Zustand in den angeregten Zustand $\mathrm{Me}^{\oplus} \mathrm{O}^{\ominus} \mathrm{Me}^{2 \oplus}$ übergeht, wobei ein Elektron vom Sauerstoffatom zum linken Metallion übertragen wird. Später sind noch verschiedene andere Mechanismen der Elektronenübertragung beschrieben worden. Eine zusammenfassende Darstellung gab Goodenough ${ }^{17}$.

Das magnetische Moment von $\mu_{\mathrm{eff}}=3,85 \mu_{\mathrm{B}}$ beruht also auf einer Elektronenübertragung infolge von indirekter Wechselwirkung. Sowohl der Antiferromagnetismus als auch die indirekte Wechselwirkung sind in der besonderen Struktur des Ferritins begründet. Die Eisen und Sauerstoff enthaltenden Mizellen gehören nach $\mathbf{F}_{\text {AnkucheN }}{ }^{5}$ zur kubischen Raumgruppe; $\mathrm{H}_{\text {ARrison }}{ }^{4}$ fand bei Ferritin A die orthorhombische Raumgruppe $\mathrm{P} 2{ }_{1} 2_{1} 2, \mathrm{P} 2_{1} 22_{1}$ oder P22 $2_{1} 2_{1}$. Die acht Ecken der Raumgruppe sind von Eisenatomen besetzt. Die Lage der Sauerstoffatome konnte nicht genau ermittelt werden. Da aber die Lage der miteinander wechselwirkenden Atome bei vielen ortho-rhombischen antiferromagnetischen Kristallen kubisch ist, ist es sehr wahrscheinlich, daß auch bei Ferritin Eisen- und Sauerstoffatome in einem kubischen Gitter abwechselnd eingebaut sind. Durch eine solche Anordnung wird jedenfalls der Antiferromagnetismus des Ferritins und die beschriebene Elektronenübertragung stark begünstigt.

Es sind verschiedene Modellvorstellungen für die Elektronenübertragung möglich. Am wahrscheinlichsten ist die Übertragung der Elektronen vom Sauerstoff zum Eisen in der folgenden Weise:

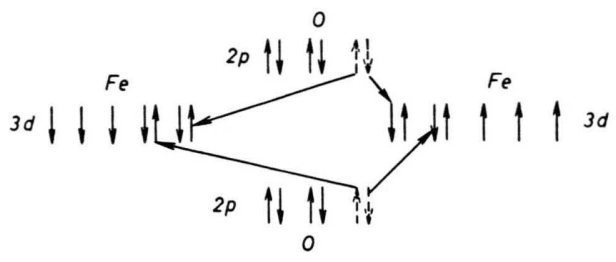

Hierbei werden für Eisen nur Elektronen auf den 3 d-Bahnen und für Sauerstoff nur solche auf den $2 p$ Bahnen berücksichtigt. Das Eisen wird dabei einwertig und der Sauerstoff nullwertig. Die Elektronenübertragung bzw. eine so weitgehende Überlappung der Elektronen-Eigenfunktionen ist nur in-

15 H. A. Kramers, Physica 1, 350 [1934].

16 P. W. Anderson, Physical. Rev. 79, 350 [1950].

17 J. B. Goodenough, Magnetism and the Chemical Bond, Interscience, New York 1963. folge der besonderen Struktur des Ferritins möglich. Nicht nur bei Antiferromagnetika, sondern auch bei Halbleitern und anderen Stoffen können jedoch mitunter sehr starke Elektronenverschiebungen innerhalb des Gitterverbandes festgestellt werden. Im vorliegenden Falle erklärt sich das reduzierte magnetische Moment von $\mu_{\mathrm{eff}}=3,85 \mu_{\mathrm{B}}$ dadurch, daß nach der Elektronenverschiebung das Eisen nur drei und der Sauerstoff gar keine ungepaarten Elektronen besitzt. Der theoretische Wert für drei un. gepaarte Elektronen beträgt $3,87 \mu_{\mathrm{B}}$. Übrigens wurden stark reduzierte magnetische Momente in antiferromagnetischen Eisenverbindungen schon mehrfach gefunden, so z. B. bei $\mathrm{FeF}_{3} \mu_{\mathrm{eff}}=1,9 \mu_{\mathrm{B}}$, bei FeSi $\mu_{\text {eff }}=2,55 \mu_{\mathrm{B}}$, bei $\mathrm{LaFeO}_{3} \mu_{\text {eff }} \sim 3 \mu_{\mathrm{B}}$ usw. Auch der stark herabgesetzte Magnetismus des $\mathrm{FeTe}_{2}$ wurde in ähnlicher Weise wie hier durch Elektronenübertragung erklärt ${ }^{18}$.

Andere Modellvorstellungen für die Elektronenübergänge sind weniger wahrscheinlich. Ein Übergang von zwei Elektronen vom Eisen zum Sauerstoff würde z. B. ebenfalls das herabgesetzte magnetische Moment erklären, aber das Eisen müßte dabei fünfwertig werden. Fünfwertiges Eisen ist zwar nicht unbekannt, z. B. im Falle des $\mathrm{K}_{3} \mathrm{FeO}_{4}$, doch sind derartige Verbindungen wenig stabil und werden durch Hydrolyse leicht zerstört. Im Falle des Ferritins erscheint es unwahrscheinlich, daß die Elektronen vom Eisen abgetrennt und an den stark elektronegativen Sauerstoff angelagert werden. Dazu wäre ein erheblicher Energieaufwand nötig und überdies müßte das die Eisen-Mizelle umgebende Eiweiß eine solche Struktur vor verschiedenen äußeren Einflüssen wirksam abschirmen.

Eine dritte Möglichkeit wäre die Übertragung zweier Elektronen von einem Eisenatom über die Sauerstoffatome zum anderen Eisenatom. Dies würde zu gleichzeitigem Auftreten von $\mathrm{Fe}^{\oplus}$ und $\mathrm{Fe}^{5 \oplus}$ führen. Eisenkomplexe mit gemischten Wertigkeiten sind ebenfalls bekannt. Die Theorie der indirekten Wechselwirkungen erlaubt prinzipiell solche Prozesse. Sie wurden erstmalig von Yamashita und Kondo ${ }^{19}$ an Manganoxyd beschrieben. Im Falle des Ferritins müßte es jedoch bei einer Konfiguration: $\mathrm{Fe}^{\oplus}\left(\mathrm{d}^{7}\right)-\mathrm{Fe}^{5^{\oplus}}\left(\mathrm{d}^{3}\right)$ zur Beimischung von Ferro.

18 J. P. Llewellyn u. T. Smith, Proc. physic. Soc. 74, 65 [1959].

19 J. Yamashita u. J. Kondo, Physical. Rev. 109, 730 [1958]. 
magnetismus kommen. Sie würde dadurch entstehen, $\mathrm{da} ß \mathrm{im} \mathrm{Fe}^{5 \oplus}$ die $\mathrm{e}_{\mathrm{g}}$-Bahnen unbesetzt sind(Konfiguration $\mathrm{t}_{2 \mathrm{~g}}^{3} \mathrm{e}_{\mathrm{g}}{ }^{0}$ ) und daß die Wechselwirkung zwischen $\operatorname{den} t_{2 g}^{3}$-Elektronen des Eisens und den $\mathrm{p} \sigma$-Elektronen des Sauerstoffs zur Paralleleinstellung der Elektronenspins führt.
Ich danke Herrn Prof. W. Buckel für die Ermög. lichung der Messungen bei der Temperatur des flüssigen Heliums. Herrn cand. phys. G. Bürк danke ich für seine Hilfe bei den Messungen und bei der Auswertung der Ergebnisse. Mein besonderer Dank gilt Herrn Prof. H. Schreiber für sein förderndes Interesse. Die Arbeiten wurden durch eine Sachbeihilfe der D e u t s c h e n Fors chungs gemein schaft unterstützt.

\title{
Freies Tryptophan in Wildform und Augenfarbmutanten von Calliphora erythrocephala während der Imaginalentwidklung
}

\author{
Helmut Langer * und Henning Grassmäder \\ Zoologische Institute der Universitäten München und Würzburg \\ (Z. Naturforschg. 20 b, 172-177 [1965] ; eingegangen am 5. November 1964)
}

\begin{abstract}
Der Gehalt an freiem Tryptophan steigt im Anfang der Puppenzeit an und hat etwa zu deren Mitte ein Maximum. Bis zu diesem Zeitpunkt sind die Konzentrationen in der Wildform und den beiden Mutanten white und chalky gleich. Bei der Normalform und den wildfarbigen Männchen der Mutante $w$ erfolgt dann - vom Einsetzen der Augenausfärbung bis zum Schlupf der Imago eine signifikante Verringerung der Tryptophankonzentration, während in der weißäugigen Mutante $c$, die keine Ommochrome bildet, die Konzentration dieser Aminosäure während der zweiten Hälfte der Puppenzeit konstant bleibt. In den Weibchen der Mutante $w$, deren Augen nur sehr wenig Ommochrome enthalten, kehrt die Tryptophankonzentration, nach Durchlaufen eines Zwischenminimums, am Ende der Puppenzeit bis fast zum Maximalwert zurück. Das nach der Zeit der Augenausfärbung überflüssige Tryptophan geben Wildform und beide Mutanten in einer ihrer Fähigkeit zur Ommochrombildung umgekehrt entsprechenden Menge mit den Meconien ab.
\end{abstract}

Bei Calliphora erythrocephala sind bisher zwei Mutationen bekannt, die die Augenfarbe beeinflussen. Beide unterdrücken die Ausbildung der Ommochrome, eine davon verhindert außerdem die Synthese der Pteridine. Bei dem Stamm $w\left(\mathrm{~T}_{\mathrm{ATE}}\right)^{1}$ haben die Weibchen eine stark verringerte Menge an Xanthommatin in den Augen, die deshalb gelborange gefärbt erscheinen, während die Augen der Männchen wildfarbig dunkelrot sind; der Pteridinbestand ist in beiden Geschlechtern etwa gleich dem der Wildform (Hanser, Ziegler $)^{2,3}$. Bei dem Stamm $c$ (Langer, Langer und Patat) ${ }^{4,5}$ haben alle Tiere rein weiße Augen wegen des völligen Fehlens von Ommochromen und Pteridinen. Die Wirkungsweise

* Ich danke der Deutschen Forschungsge. $\mathrm{me}$ in s chaf $\mathrm{t}$ für die Unterstützung dieser Untersuchungen.

1 P. Tate, J. Genetics 48, 176 [1947].

2 G. Hanser, Z. Naturforschg. 14 b, 194 [1959].

3 I. Ziegler, Z. Vererb.-lehre 92, 239 [1961].

4 H. Langer, Nature [London] 194, 111 [1962].

5 H. Langer u. U. Patat, Verh. dtsch. Zoolog. Ges. 55, 174 [1962].

6 M. M. Green, Genetics 34, 564 [1949].

7 R. Danneel u. B. Zimmermane, Z. Naturforschg. 9 b, 788 [1954]. beider Gene ist noch nicht geklärt. Da die Synthese der Ommochrome vom Tryptophan ausgeht, ist bei der Analyse der Genwirkung in ähnlichen Mutanten anderer Insekten der Gehalt an Tryptophan und seinen Folgeprodukten untersucht worden (Drosophila: Green, Danneel und Zimmermann, Shapard, Wessing und Danneel; Ephestia: Caspari, Caspari und Richards, Butenandt und Albrecht, Egelhaaf, Plodia: De Almeida) ${ }^{6-9 ; 10-14 ; 15}$. Für Calliphora liegt eine Angabe von Sсотт ${ }^{16}$ über den Gehalt an freiem Tryptophan während der Entwicklung der Wildform vor.

Die sichtbare Ablagerung von Xanthommatin in den sich entwickelnden Facettenaugen setzt bei Calli-

8 P. B. Shapard, Genetics 45, 359 [1960].

9 A. Wessing u. R. Danneel, Z. Naturforschg. 16 b, 388 [1961].

10 E. Caspari, Genetics 31, 454 [1946].

11 E. Caspari u. J. Richards, Proc. nat. Acad. Sci. USA 34, 578 [1948].

12 A. Butenandt u. W. Albrecht, Z. Naturforschg. 7 b, 287 [1952].

13 A. Egelhaaf, Z. Naturforschg. 12 b, 465 [1957].

14 A. Egelhaaf, Z. Vererb.-lehre 94, 349 [1963].

15 F. Ferrand de Almeida, Naturwissenschaften 48, 27 [1961].

16 T. A. Sсотт, PhD-Thesis, Univ. of Leeds (England), 1960. 